\title{
Quantum Chemistry Applied to Photocatalysis with $\mathrm{TiO}_{2}$
}

\author{
Sergio Ricardo de Lazaro, \\ Renan Augusto Pontes Ribeiro and \\ Luis Henrique da Silveira Lacerda
}

Additional information is available at the end of the chapter

http://dx.doi.org/10.5772/intechopen.69054

\begin{abstract}
Heterogeneous catalysis is a topic very studied in science. Its application in technologies of energy conversion, water purification, chemical synthesis, car catalytic converter and so on is studied. Recently, the $\mathrm{TiO} 2$ material in anatase and rutile phases has been used extensively in photocatalytic systems; its band-gap is localized in visible and ultra-violet spectra, proportioning a good material for generation of chemical radicals. Nowadays, the density functional theory (DFT) is shown as a great tool to simulate all types of materials and the possibilities to simulate bulk and surfaces of materials importance in last few decades. Recently, quantum periodic calculations based on DFT methods have been widely used to simulate materials and the main functionals applied are PBE, PBE0 and B3LYP; they are important for doping and adsorption theoretical investigations and are present in various simulation programs, such as, Crystal, Wien, Vasp and others. This methodology has investigate the influence of dangling bonds, cationic and anionic doping, charge transfer, surface energy and more quantum properties. Quantum chemistry tools, in particular, DFT methods, are key points to develop high quality research and technology once theoretical calculations are important to guide and understand the molecular design in photocatalysis.
\end{abstract}

Keywords: solid state, chemistry of materials, simulation, electronic structure, bulk, surface, optical property 


\section{Introduction}

Over the last few decades, the field of photocatalysis has assumed a remarkable importance in environmental topics, mainly on technologies based on the capability of use of the main supply of energy on Earth: solar irradiation. In this case, the solar light can be used to activate a chemical process of radicals species, destroy undesired compounds or be transformed in chemical energy; for example, hydrogen production from $\mathrm{H}_{2} \mathrm{O}[1,2]$. The aim of this field was initially motivated by the oil crisis, which promoted the research about alternative energy sources. In addition, the concern about pollution has attracted a recent economical, political and scientific interest because of the toxicological potential of recalcitrant compounds for environmental contamination, as well as for animal and human health. Thus, eco-friendly methods became a fundamental topic, being the advanced oxidation processes (AOPs) the most powerful tool to destroy recalcitrant synthetic species due to high reactive potential of photoinduced radicals, which allows the extinction of a wide range of compounds $[1,3,4]$.

Nowadays, heterogeneous photocatalysis proved its efficiency to degrade chemical contaminants through a photoinduced reaction in the presence of a semiconductor photocatalyst. This method was originated in the decade of 1970 when Fujishima and Honda described the water splitting by photoelectrochemical cell containing $\mathrm{TiO}_{2}$ [5]. Posteriorly, the scientific and technological interest in such methods has exponentially increased, $\mathrm{TiO}_{2}$ being the widely used semiconductor material for charge carriers generation due to the photostability, chemical and biologically inert nature, availability and low cost. Such material can be found/synthetized in anatase, rutile and brookite phases, anatase being a polymorph, the most prominent candidate for photocatalytic applications. Anatase $\mathrm{TiO}_{2}$ is an n-type semiconductor with indirect band gap of around $3.2 \mathrm{eV}$. Several reasons were proposed to explain the higher photocatalytic efficiency of anatase polymorph, such as increased surface area, $-\mathrm{OH}$ group concentrations and electronic structure features. In particular, the electronic features have been shown as the key advantages of anatase $\mathrm{TiO}_{2}$ mainly related to the band edge positions, which allow the reduction or oxidation of protons from water creating $-\mathrm{OH}$ radicals as intermediate in the photo-oxidation reactions to break organic compounds because of high oxidation potential $[1,6,7]$.

However, due to large band gap $(3.2 \mathrm{eV})$, anatase $\mathrm{TiO}_{2}$ absorbs only UV light above $400 \mathrm{~nm}$, which amounts for $4-5 \%$ of the solar photons. To enable the effective use of solar radiation in photocatalytic process with $\mathrm{TiO}_{2}$, much effort has been directed to the narrowing of band gap from UV to the visible spectra range. Such efforts include the crystal shape engineering and the doping or co-doping process. Recently, a lot of theoretical and experimental studies have been developed to investigate metals ( $\mathrm{Fe}, \mathrm{Cr}, \mathrm{V}, \mathrm{Mo}, \mathrm{Re}, \mathrm{Ru}, \mathrm{Mn}, \mathrm{Co}, \mathrm{Rh}$ ) and non-metals (N, S, B, C, F) doping effects on electronic structure of anatase $\mathrm{TiO}_{2}$ photocatalysts [7-9]. In the metal doping process, the theoretical and experimental reports suggest that a redshift of the band gap occurs due to the insertion of a new electronic band closer to the conduction band minimum (CBM) improving the photocatalytic properties. Despite narrowing of the band gap, foreign cations frequently act as recombination center, suggesting that significant improvements in the photocatalytic efficiency are possible only at low concentration of dopants [10-12]. Alternatively, the coupling of $\mathrm{TiO}_{2}$ with another semiconductor $\left(\mathrm{SnO}_{2}, \mathrm{WO}_{3}, \mathrm{CdS}\right.$ and others) is commonly 
used as an approach to improve the photocatalytic efficiency through the band structure mismatch, which allows a physical separation between the photoinduced charge carriers, reducing the recombination rate. On the other hand, for non-metal doping, there are three different main opinions about the band-gap narrowing that can be related to the shift of valence band maximum (VBM), creation of impurity levels or oxygen vacancies [7-9].

Therefore, the main challenge in titania-assisting photocatalytic process remains on the tailoring of their electronic structure in order to improve and stabilize the photoinduced reactions. From a lot of experimental and theoretical researches, it is possible to investigate the understating of the main features of these complex reactions, such as the control and engineering of band gap, band edges, charge transport and recombination rate. In this aspect, theoretical analyses are helpful due to the precision of quantum mechanics-based methods to predict structural-electronic properties and its changes from chemical compounds. For example, recently it was published in manuscripts announcing the reliability of density functional theory (DFT) methods to research the band structure distribution, band-gap evaluation and engineering, electron-hole transport and dielectric constant, which are key properties of semiconductor photocatalytics. Besides the theoretical endeavor to investigate and predict bulk, surface chemistry, electronic structure and other properties have played a fundamental role in the understanding of morphological transformations and crystal growth, as well as for adsorption and reaction phenomena [13-15].

In this chapter, we propose a theoretical point of view about the photocatalytic properties of $\mathrm{TiO}_{2}$, focusing on the evaluation of electronic structure parameters for both bulk and surfaceoriented materials. The following sections are dedicated to explain (i) the mechanism of titaniaassisted photocatalytic properties, (ii) benchmark of DFT methods on the investigation of photoinduced properties and (iii) theoretical results and discussion for different $\mathrm{TiO}_{2}$ models.

\subsection{Basic mechanism of $\mathrm{TiO}_{2}$ photocatalysis}

Photo-driven processes, such as used in photocatalytic and photovoltaic devices, are based on the conversion of light energy into other forms of energy such as electricity (solar cells) or chemical compounds (photocatalysis, water splitting, $\mathrm{CO}_{2}$ reduction and others). These technologies, mainly photocatalytic processes, require the semiconductor electronic structure for the light absorption and conduction of the photoinduced charge carriers. In a molecular point of view, the general mechanism behind such devices can be divided in three steps: (i) light adsorption; (ii) electron-hole dissociation and (iii) charge carriers dynamic [1, 2, 13].

In the first step, commonly described as light adsorption, the light interacts with the electronic structure of the semiconductor. In this case, the light wavelength must be equal to or higher than the band gap, the energy difference between $V B$ and $C B$, to promote an electron $\left(\mathrm{e}^{-}\right)$from the VB to $\mathrm{CB}$, inducing an electronic vacancy in the VB, denominated as hole $\left(\mathrm{h}^{\bullet}\right)$. The electronhole pair interacts through a Coulomb attraction and plays a fundamental role in subsequent steps, controlling the photocatalytic efficiency of semiconductors. Especially for sunlight absorption, an optimum band gap is required due to the light wavelength commonly found in solar radiation. For the solar-driven photocatalysis, the optimum band gap belongs to the range between 1.6 and $2.5 \mathrm{eV}$. In addition, for photoinduced reactions, another compromise 
needs to be achieved: besides the band-gap value, the energy of the electron/hole should be high enough to perform the given reactions. These chemical potentials depend on the position of energy levels in the semiconductor, which is one of the key advantages of $\mathrm{TiO}_{2}$ among other semiconductors because both the reduction of protons $\left(\mathrm{E}_{\mathrm{NHE}}\left(\mathrm{H}^{+} / \mathrm{H}_{2}\right)=0.0 \mathrm{ev}\right)$ and the oxidation of water $\left(\mathrm{E}_{\mathrm{NHE}}\left(\mathrm{O}_{2} / \mathrm{H}_{2} \mathrm{O}\right)=1.2 \mathrm{ev}\right)$ can be activated simultaneously. Moreover, the superficial $-\mathrm{OH}$ groups can act as donor species to generate $\mathrm{OH}^{\bullet}$ radicals that have a very high oxidation potential, which enables the subsequent reactions used in chemical decontamination $[1,6,9,13]$.

Another important feature associated with the optical excitation corresponds to the band-gap nature, which plays an important role in the absorption coefficient of the semiconductor. For semiconductors the band gap can be direct or indirect, depending upon the localization of the $\mathrm{VBM}$ and $\mathrm{CBM}$ along the Brillouin zone. In photocatalysis, the band-gap nature is important in the recombination of photo-generated electrons and holes due to differences in the electron decay from CBM. For semiconductors with direct band gap, such as rutile $\mathrm{TiO}_{2}$, the recombination of the charge carriers emits a photon once the CBM and VBM are located in the same $k$ vector. However, for indirect band-gap semiconductors such as anatase, the recombination is assisted by a phonon due to the difference between CBM and VBM, making the direct recombination difficult between excited electrons and holes, which results in an increased electronhole lifetime. As a key result, the diffusion rate and the reaction time of the excited electron hole in indirect semiconductor also increase, making them promising candidates with superior photocatalytic performance than direct semiconductors [16, 17].

In the next step, the electron and hole have to be dissociated to obtain free charge carriers, which will be used in the electron transport of the device. This dissociation depends on the electron-hole binding energy $\left(E_{\mathrm{b}}\right)$ that is inversely proportional to the semiconductor dielectric constant. If we assume that the electron-hole dissociation will be made by the thermal energy, $E_{\mathrm{b}}$ should be lower than $k_{B} T\left(k_{B}=\right.$ Boltzmann constant and $T=$ absolute temperature) around $25 \mathrm{meV}$ at room temperature, and the semiconductor must have a dielectric constant around 10. For $\mathrm{TiO}_{2}$, both rutile and anatase polymorphs have a superior dielectric constant, which enables a lower binding energy between the electron holes, making the dissociation easier $[13,16]$.

The final step corresponds to the diffusion of free charge carriers. In this step, the electron and the hole are transported to their active sites where they will be used before the recombination $[1,6,13,16]$. The diffusion $(D)$ is strictly related to the mobility $(\mu-$ Eq. $(1))$ of the charge carrier which in turn is linked to the effective mass $\left(m^{*}\right)$ and the collision time $(\tau)$ of the charge carrier (Eq. (2)). Therefore, $D$ is increased if the effective mass of the photogenerated carriers becomes lighter resulting in enhanced photocatalytic efficiency. Furthermore, the ratio between the effective mass of electrons $\left(m_{\mathrm{e}}{ }^{*}\right)$ and holes $\left(m_{\mathrm{h}}{ }^{*}\right)$ is a powerful tool to predict the electron/hole pair stability with respect to the recombination process. In this case, a larger effective mass difference induces distinct mobility, which reduces the electron-hole pair recombination, increasing the photocatalytic efficiency [13, 14, 17, 18].

$$
D=\frac{k_{B} T}{e}
$$




$$
\mu=e \frac{\tau}{m}
$$

However, the simulations involving scattering process are very expensive and the evaluation of mobility of charge carriers requires an alternative approach. The effective mass can be associated with the band curvature at the top of the VB or at the bottom of CB. For a single isotropic and parabolic band, the effective mass can be obtained through the expression:

$$
\frac{1}{m}=\frac{1}{\hbar} \frac{\partial^{2} E}{\partial k^{2}}
$$

Therefore, $m_{\mathrm{e}}{ }^{*}$ can be obtained by fitting the bottom of the conduction band, whereas $m_{\mathrm{h}}{ }^{*}$ corresponds to the fitting along the top valence band. In order to acquire the validity of the parabolic approximation within the CBM and VBM regions, the parabolic fitting must be done within an energy difference around $26 \mathrm{meV}$ near to the CBM and VBM, corresponding to the thermal dissociation energy of carriers at room temperature $[18,19]$.

\section{Theoretical methods and density functional theory}

The theoretical methods based on quantum mechanical simulations are an important tool to evaluate material properties, mainly at the molecular level. Historically, the development of new materials to technological applications appears as a difficult task which requests a long time of studies. Meanwhile, the theoretical-computational method plays the traditional role of study of materials already discovered. However, the technological advancement is extremely dependent on development of new materials, once such materials are responsible for the improvement of available technologies. Front of such need, the theoretical methods helped chemists and physicist on the development materials at higher speed; it was possible once the theoretical investigation provides the materials characteristic and the limitations to its applications [20-22]. A large number of computational methods is available for investigation of material properties; however, in the last three decades, the use of DFT has changed the world because it offers an excellent relation between results precision and calculation time. The importance of this theory on material investigation is evidenced by the number of manuscripts based on its application since is very superior than the number of manuscripts based on Hartree-Fock (HF) and semi-empirical methods simulations as for inorganic chemistry as for organic compounds [20,21,23].

\subsection{Density functional theory}

The material properties can be evaluated through several computational approaches, such as molecular dynamics, ab initio methods and semi-empirical methods. The calculations based on molecular dynamics evaluated the system properties based on the behavior of ball-and-springs model under application of a force external field to atoms representation. In turn, the $a b$ initio and semi-empirical methods employ different approaches to solve the Schrödinger Equation and for the obtainment of system wave function ( $\Psi$ ) [24]. Particularly, the DFT system inter- 
pretation is not based on wave-function $(\Psi)$, once it assumes the system total energy as a single functional of electronic density $(\rho)$. Poorly, this theory can be simplified in two basic postulates [24, 25]:

i. The density functional $(\rho)$ determines exactly and completely all the ground state properties for a system. Thus, $\mathrm{Q}$ is only dependent on three variables that determine the position $(x, y$ and $z)$;

$$
\rho_{(x, y, z)}=E_{0}
$$

ii. Any function for electronic density will have energy greater than or equal to the ground state energy for a real system.

$$
E_{(v)}\left[\rho_{0}\right] \geq E_{(0)}\left[\rho_{0}\right]
$$

Nevertheless, the analytical function for electronic density is not yet known and the electronic density is obtained by HF equations for achievement of $\varrho$ by a self-consistent field (SCF) method. Hence, the HF method is very similar to DFT so that the difference lies in the equation's formalism. Although this similarity was observed, the DFT shows highest precision and low time (computational cost) regarding HF simulations due to number of variables in each methodology. The HF and semi-empirical methods employ a high number of variables for a system investigation; the number of variables is in the $4 n$ order, where $n$ refers to the number of electrons in the system. In turn, DFT is dependent on three variables [20, 22, 24, 25].

Actually, the quantum calculation based on DFT applied the theorem developed by Kohn and Shan (KS) in 1965; the KS equation describes all the functional theories (Eq. (6)) and their representation for molecular orbitals (Eq. (7)). In such equations, $\nabla^{2}$ is the kinetic energy for non-interacting electrons; $u(r)$ refers to classic Coulomb potential for a density of $n$ electrons; $d$ refers to the system space and $\varphi$ corresponds to molecular orbital [26]. The KS equation was applied on two different systems; the first considers that there are no interactions between electrons, whereas the other assumes that such interactions are observed. The obtained results for both systems indicate a significant difference of energy between them. In order to correct this difference, the exchange-correlation term $\left(E_{\mathrm{XC}}\right)$ was inserted in DFT formulism; the $E_{\mathrm{XC}}$ consists of the sum of kinetic and potential energy difference between interacting and non-interacting systems. In terms of system interpretation, this energy refers to $1 \%$ of system total energy, and its physical meaning is the interaction between electrons in the investigate compound. Thus, DFT describes $100 \%$ of system total energy.

$$
\begin{gathered}
E_{K S}=-\frac{1}{2} \sum_{i} \int d^{3} r \phi_{i}^{*}(r) \nabla^{2} \phi_{i}(r)+\int d^{3} r\left[\frac{1}{2} u(r)+V_{e x t}(r)\right] \rho(r)+E_{X C}[\rho] \\
{\left[-\frac{1}{2} \nabla^{2}+V_{e f f}(r)\right] \phi_{i}(r)=E_{i}^{K S}(r) \phi_{i}(r)}
\end{gathered}
$$


The main characteristic of $E_{\mathrm{XC}}$ is the possibility of description by several forms dependent on what exchange-correlation functional was employed. Thereby, the choice of a functional to describe $E_{\mathrm{XC}}$ has a giant effect on material properties evaluation, as offering better results and as offering a reduction in computational cost. The first functionals are called local functionals and are based on an electron cloud model to represent the system electronic density; among its class of functionals, stands out the local density approximation (LDA)/local spin density approximation (LSDA) and generalized gradient approximation (GGA) [20, 22, 25, 27, 28]. Further ahead, the use of hybrid functionals gains force due to high proximity to experimental results.

\subsubsection{Exchange-correlation functionals}

The $E_{\mathrm{XC}}$ term can be described in several forms according to the employed functional. Recently, the functionals are classified as local, non-local and hybrid functionals. Among the local approximations, stands out the LDA that is a general approach to represent the electron gas model and offers a simple description of exchange correlation at local character. The employment of LDA functional results in exact values for system kinetic energy shows better results when applied to a system where $\rho$ slowly changes similar to a uniform electron gas. It is observed because of the LDA evaluates a real system, in which electronic density is $E_{[\rho]}(r)$, by a homogeneous electrons gas system with the same density. Although such representation is valid, the density $\rho$ becomes independent of position since the electrons are evenly distributed in the system. Hence, the investigation of real system by LDA functional is not accurate and over-estimates the correlation energy at $100 \%$. The non-local functionals are developed in order to correct this failure [24, 25]. Furthermore, the LSDA is also a local description of the $E_{\mathrm{XC}}$ term and is very similar to LDA; both functionals are based on electron gas model but the LSDA includes the electron spins to system properties determination [20, 24].

The non-local functionals were developed aiming to correct the LDA failures in representation of real system. These approaches are based on a charge gradient and are also commonly known as corrected gradient functional. Among the non-local approximations, stands out the GGA which uses the electronic density gradient; this gradient consists of the first derivative of $\mathrm{Q}$ as a function of its position. The GGA functional obtains the $E_{\mathrm{XC}}$ terms by the sum of correlation energy $\left(E_{\mathrm{C}}\right)$ and exchange energy $\left(E_{\mathrm{X}}\right)$, both negative values. The GGA functionals offer better results than LDA and LSDA approximations since the relation between $\varrho$ and position is considered. Compared to LDA and LSDA approximations, the GGA shows a deviation of $1 \%$ for system $E_{\mathrm{XC}}$ once the electronic density distribution is treated in real form and not as an electron gas model [25, 29, 30]. However, the results presented by GGA approximations can be improved from Correlations (Becke Functionals) and Exchange Functionals (LYP and P86) [24].

Ultimately, the other class of functionals is known as hybrid functionals. Such functional employs Hartree-Fock method parameters to determine the $E_{X C}$ energy. The first hybrid functional was the half-half functional which was developed by Becke, and it was developed by a linear description of electronic density. However, this functional shows several limitations. Thus, in 1993, Becke proposes the B3PW91 model that uses three empirical parameters 
( $a=0.20, b=0.72$ and $c=0.81$ ) to adequate the theoretical results to experimental results combined to Perdew and Wang correction gradient (PW91). This new functional has excellent results compared to half-half functional due to its more precise description of $E_{X C}$ energy and parameterization $[21,27,28,31,32]$.

In 1994, Frisch and co-authors adapted the LYP corrections rather than PW91 creating the B3LYP that uses the same empirical parameters of B3PW91. Such functional employs the Slater exchange plus Vosko, Wilk, Nusair (SVWN) to improve the correction proposed by LYP. In the last few years, B3LYP has become one of the most used for computational calculations because of the excellent results obtained. Another factor responsible for the wide use of hybrid functional is its versatility, and once such functional was used to investigate semiconductors, proteins, organic compounds and others [20, 21, 24, 26-28, 31-35].

The hybrid functionals are differentiated according to the percentage of parameters of the Hartree-Fock method employed in the determination of $E_{X C}$. For example, B3LYP uses 20\% of HF parametrization in its formalism. In 1996, Perdew and co-workers increased the HF percentage to $25 \%$ in PBE functional and created the PBE0 functionals. The increase in HF aims to minimize the over-estimation of factors arising from the electrons interaction; the PBE0 uses the GGA to evaluate the $E_{\mathrm{XC}}$ energy and it does not present adjustable parameters in its formulation (empirical parameters are defined as $a=0.25, b=0.75$ and $c=1$ ). Other differences regarding B3LYP is less sophisticated formulation to PBE0 [27, 36, 37]. Moreover, PBE0 has great efficiency in material properties determination.

Another functional that is largely used is the HSE which was developed by Heyd, Scuseria and Ernzerhof. This functional investigates the $E_{\mathrm{XC}}$ energy by slitting it in two parts: a short-range part and a long-range part. Particularly, the HSE hybrid functional employs the parameter $\omega$ in function of Bohr radius. Thus, the short-range part is treated in the same form as that in the PBEh global hybrid, that is, it uses $25 \%$ short-range exact exchange and $75 \%$ short-range PBE exchange; whereas, the long-range part is investigated by PBE. If $\omega$ goes to 0 , the short-range part dominates and HSE reduces to PBEh; if $\omega$ goes to infinity, the short-range part disappears and HSE reduces to PBE. The use of HSE functional reduces the computational cost to obtain the exact exchange-correlation energy [38].

\section{Materials modeling based on DFT}

The employment of DFT on material modeling offers an excellent relation time $\times$ efficiency. The efficiency is denoted by the results precision to prevision of material properties. In case of photocatalysis applications of solid-state materials, a theoretical methodology based on DFT provides important information about band gap, band-gap nature, photoinduced behavior, density and mobility for charge carriers; thus, it is possible to determine the applicability of each material in photoinduced devices and processes.

In order to evaluate these properties, it is necessary to investigate the material electronic structure. In solid-state materials, the electronic structure sorts materials as insulators, conductors and semiconductors. The formation of electronic structure of solid-state materials is 
originated from interactions between all constituent atoms since the atomic energy levels are perturbed by the neighbor atoms. Thereby, in these materials, the overlap of a large number of valence atomic orbitals results in the formation of molecular orbitals with very close energy, forming a quasi-continuous band; that is, an energy band is a continuum of closely spaced electron states [39-41]. Each energy band is occupied by two electrons ( $\alpha$ and $\beta$ ) as predicted by Pauli exclusion principle. In solid-state materials, the electrons are observed in the lowest energy states; the last occupied energy level refers to the top or VBM, whereas the first unoccupied energy level is the bottom or CBM. There is no electronic state in the region between the VBM and CBM and it is called the band-gap which has direct influences on conduction properties of a solid [42]. According to band-gap energy, a material is classified as conductor (band gap > $1.0 \mathrm{eV}$ ), semiconductor (band gap from 1.0 to $4.0 \mathrm{eV}$ ) and insulator (band gap $4.0 \mathrm{eV}$ or higher) [40, 43].

\subsection{Density of states (DOS) and band structure}

The DOS analysis (Figure 1) consists of a graphical representation of packing level of energy states in a quantum system, that is, the number of states in each region of energy. A high amount of energetic states results in a high density of states on projected DOS. In general, the DOS analysis is employed to investigate the energy levels nearest to band gap. As observed in Figure 1, the band of lowest energy refers to valence band and the higher energy band is the conduction band. Besides, the DOS analysis can also provide the contribution of each atom to compose the valence and conduction bands; by contribution of this analysis, it is also possible

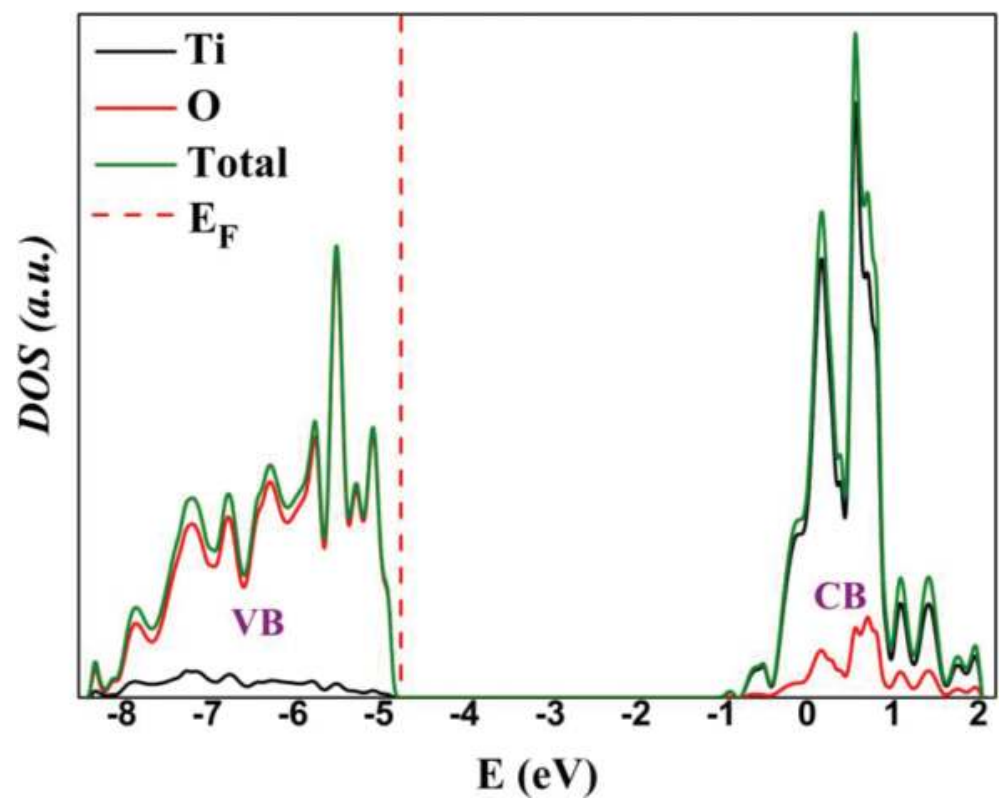

Figure 1. Projected DOS on $\mathrm{Ti}$ and $\mathrm{O}$ atoms of anatase $\mathrm{TiO}_{2}$. The region before Fermi energy $\left(E_{\mathrm{F}}\right)$ is the valence band and the region after $-1 \mathrm{eV}$ is the conduction band. 
to clarify the chemical bond composition and predict the electronic configuration of atoms [4043]. Furthermore, applying the one-third of Simpson rule [44] on projected density of states for pure and doped models, it is possible to evaluate the number of available states in VB and CB. Such methods consist of numeric integration of the area under the DOS curve. Then, the number of states obtained from numeric integration is divided by unit cell volume. The values for $\mathrm{CB}$ and $\mathrm{VB}$ represent the number of available states for the formation of holes and electrons in electronic structure, respectively. According to the number of available states, a semiconductor material is classified as p-type (greater number of holes) or n-type (greater number of electrons) [44].

However, the energy bands for solid materials are not regular in all crystalline structures, and the same band can show a different energy level at each high symmetry point of Brillouin zone. Such symmetry points vary according to the spatial group of the crystalline structure and are labeled to their coordinates in space. The band structure analysis provides the energy of bands and the position of VBM and CBM. Thus, the band structure evaluation offers the band-gap nature to a material, that is, if the electron excitation occurs directly or indirectly [40]. Figure 2 presents the band structure for anatase $\mathrm{TiO}_{2}$; it is observed as an indirect band-gap and that the bands are not regularly distributed at high symmetry points.

The band structure study can also provide the effective mass for a solid-state material. In general, the transfer rate of electron-hole pair is inversely proportional to their effective mass. Thereby, a great effective mass denotes a low transfer rate of carriers, whereas, a small effective mass indicates that the charge carriers are extremely stable. The stability for these carriers also promotes the migration of electron and holes, as well as inhibits their recombination. The

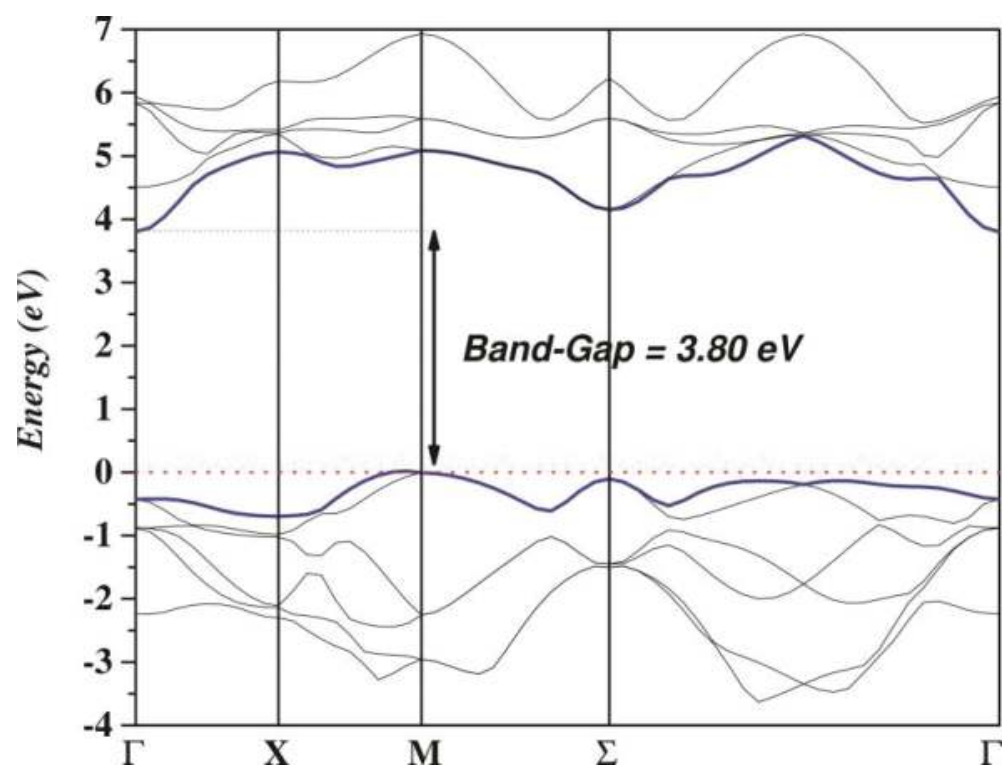

Figure 2. The band structure analysis for anatase $\mathrm{TiO}_{2}$ shows an indirect band-gap. 
effective mass of electrons and holes allows to assess indirectly the rate of charge carriers as presented in Eq. (8); in this formula, $m^{*}$ is the effective mass of the charge carrier, $k$ is the wave vector, $\hbar$ is the reduced Planck constant and $v$ is transfer rate of photo-generated electrons and holes. Besides, the effective mass for electrons $\left(m_{e^{*}}\right)$ and holes $\left(m_{h^{*}}\right)$ should be investigated regarding the CBM and VBM; such points are very important to determine the $E_{\mathrm{g}}$ for a material. The values for effective mass were calculated by a fitting parabolic function around these points (Eq. (9)); in such an equation, $m^{*}$ is the effective mass of the charge carrier, $k$ is the wave vector, $\hbar$ is the reduced Planck constant and $E$ refers to the energy of an electron at wave vector $k$ in that band. In order to guarantee the validation of the parabolic approximation within the CBM and VBM, the parabolic fitting is performed considering a difference of 1 meV around the CBM and VBM regions [45-48].

$$
\begin{gathered}
v=\hbar k / m^{*} \\
m^{*}=\hbar^{2}\left(\frac{d^{2} E}{d k^{2}}\right)^{-1}
\end{gathered}
$$

\subsection{Photoinduced properties}

The photoinduced properties of a solid compound determine how it interacts with electromagnetic radiation. In case of solid-state materials, a material interacts only with radiation which has energy equal to or higher than the material band gap [40,41]. Thus, through evaluation of $E g$ values, it is possible to determine the characteristic type of radiation that interacts with each investigated material. Allied to the deep electronic structure investigation, it is possible to determine the bulk and surfaces of photoinduced behavior of materials and its applicability on photocatalytic process. Such a condition is essential to form the electron-hole pair $\left(\mathrm{e}^{-}-\mathrm{h}^{\bullet}\right)$, which is the precursor to start the photocatalysis process. Another point is focused on energetic stability of $\mathrm{e}^{-}-\mathrm{h}^{\bullet}$ pair, and this factor can be estimated by reduced mass; however, the half-life time is not provided.

A practical example and much discussed in literature is comparing the electronic structures of rutile and anatase $\mathrm{TiO}_{2}$ to clarify these concepts. Analyzing the band structures of both materials, we found band gaps of 3.50 and $3.80 \mathrm{eV}$ (Figure 3), respectively. The minor band gap for anatase structure shows a photoinduction of $\mathrm{e}^{-}-\mathrm{h}^{\bullet}$ pair from wavelength close to 326 $\mathrm{nm}$, whereas, for rutile polymorph the $\mathrm{e}^{-}-\mathrm{h}^{\bullet}$ pair is photogenerated around to $354 \mathrm{~nm}$. Such difference is possible because of the major disorder associated with the $\mathrm{TiO}_{6}$ cluster of anatase phase in relation to rutile structure; the displacement of $\mathrm{Ti}$ atom from central position of the unit cell in anatase causes modification in electronic structure through overlap between oxygen $2 p$ orbitals and titanium $3 \mathrm{~d}$ orbitals. This overlap orients to $\mathrm{TiO}_{6}$ cluster to a $\mathrm{TiO}_{5}$ cluster configuration, while, in rutile phase this effect is smaller. However, the anatase $\mathrm{TiO}_{2}$ has an indirect band gap and rutile $\mathrm{TiO}_{2}$ shows a direct band gap indicating that for anatase the $\mathrm{e}^{-}-\mathrm{h}^{\bullet}$ pair recombination process is less possible because it requires a photon-phonon coupling and in rutile structure such phenomenon is direct, that is, localized in the same symmetric region. Thus, for anatase structure as photoinduction energy required to make the $\mathrm{e}^{-}-\mathrm{h}^{\bullet}$ pair as possibility of its recombination are more favorable than rutile phase. Now, let us evaluate the 
a)

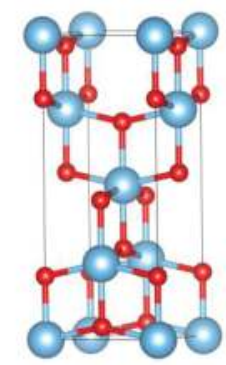

b)

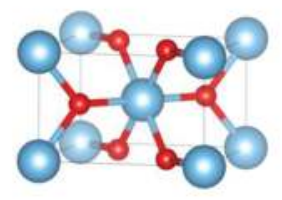

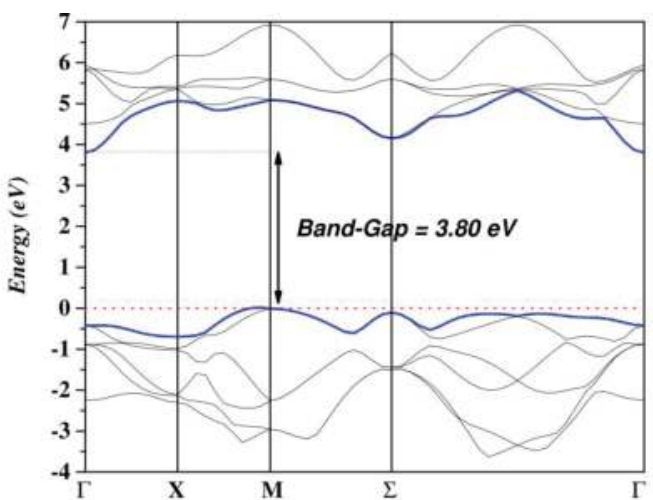

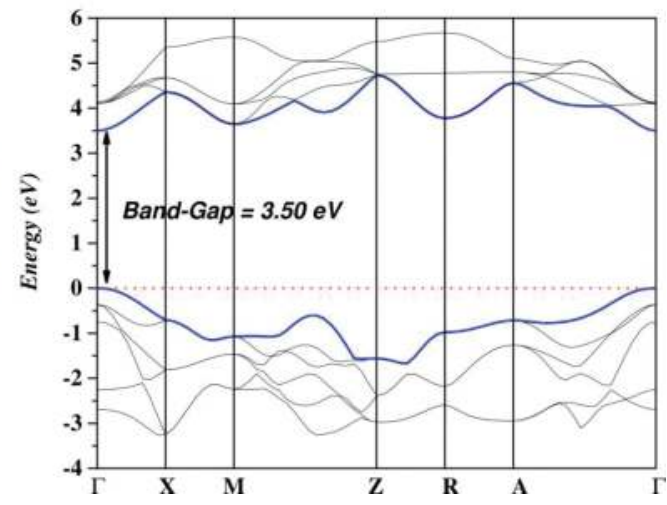

Figure 3. The crystalline structure and band structure for titania materials. (a) anatase crystalline phase and (b) rutile crystalline phase.

energetic stability of the $\mathrm{e}^{-}-\mathrm{h}^{\bullet}$ pair photoinduced through effective mass. The effective mass for electron and hole charge carriers calculated for anatase phase was 0.46 and 0.14 , respectively; then, the electron/hole ratio is 3.29 , indicating that $\mathrm{e}^{-}-\mathrm{h}^{\bullet}$ pair is favorable in anatase phase as carriers. Nevertheless, for rutile structure, this effective mass for electron and hole carriers is 0.65 and 0.32 , respectively; likewise, the electron/hole ratio is 2.03 showing that the $\mathrm{e}^{-}-\mathrm{h}^{\bullet}$ pair is less favorable than anatase structure. Therefore, every electronic structure of anatase $\mathrm{TiO}_{2}$ material from its crystalline structure is more inclined to photocatalytic process in comparison to rutile $\mathrm{TiO}_{2}$ polymorph because anatase structure has small and indirect band gap and the $\mathrm{e}^{-}-\mathrm{h}^{\bullet}$ pair is more favorable.

\subsection{Surface properties}

Surface chemistry is an interesting and challenging topic in photocatalytic applications. In this case, the molecular level can be described by the combination between the surface structure and the interface region, where the main reactions involving photoinduced charge carriers occur. Therefore, the understanding of surface chemical reactions plays a fundamental role in 
the description of chemical bond between surface and adsorbed molecules, which are the fundamental basis to clarify the material photocatalytic activity [14, 15].

Recently, a lot of theoretical studies show the benchmark of DFT methods to reproduce the main effects behind the catalytic behavior of transition metal surfaces $[49,50]$. For example, Cheng and co-workers systematically investigate the water splitting along rutile (110) surfaces combining DFT and molecular dynamics [51]. In addition, Migani and Blancafort studying the photocatalytic oxidation of methanol on $\mathrm{TiO}_{2}$ (110) surfaces trough DFT/HSE06 calculations highlighted the role of excitons to produce formaldehyde [52]. Several related reports have appeared recently in the literature, as described by Nolan and co-authors that summarize the main advances in ab initio-based design of photocalytic surfaces [53].

\section{Author details}

Sergio Ricardo de Lazaro*, Renan Augusto Pontes Ribeiro and Luis Henrique da Silveira Lacerda

*Address all correspondence to: srlazaro@uepg.br

Department of Chemistry, State University of Ponta Grossa, Ponta Grossa, Brazil

\section{References}

[1] Gaya UI, Abdullah AH. Heterogeneous photocatalytic degradation of organic contaminants over titanium dioxide: A review of fundamentals, progress and problems. Journal of Photochemistry and Photobiology C: Photochemistry Reviews. 2008;9:1-12. DOI: 10.1016/j.jphotochemrev.2007.12.003

[2] Ravelli D, Dondi D, Fagnoni M, Albini A. Photocatalysis. A multi-faceted concept for green chemistry. Chemical Society Reviews. 2009;38:1999-2011. DOI: 10.1039/b714786b

[3] Fox MA, Dulay MT. Heterogeneous photocatalysis. Chemical Reviews. 1993;93:341-357. DOI: $10.1021 / \mathrm{cr} 00017 \mathrm{a} 016$

[4] Imamura K, Kominami H. Synthetic applications of titanium(IV) oxide-based photocatalysts. In: Colmenares JC, Xu Y-J, editors. Heterogeneous Photocatalysis: From Fundamentals to Green Applications. Berlin, Heidelberg: Springer; 2016. pp. 283-320. DOI: 10.1007/978-3-66248719-8_9

[5] Hashimoto K, Irie $\mathrm{H}$, Fujishima $\mathrm{A}$. $\mathrm{TiO}_{2}$ photocatalysis: A historical overview and future prospects. Japanese Journal of Applied Physics. 2005;44:8269. DOI: 10.1143/JJAP.44.8269

[6] Carp O, Huisman CL, Reller A. Photoinduced reactivity of titanium dioxide. Progress in Solid State Chemistry. 2004;32:33-177. DOI: 10.1016/j.progsolidstchem.2004.08.001 
[7] Hernández-Alonso MD, Fresno F, Suárez S, Coronado JM. Development of alternative photocatalysts to $\mathrm{TiO}_{2}$ : Challenges and opportunities. Energy \& Environmental Science. 2009;2:1231. DOI: 10.1039/b907933e

[8] Di Valentin C, Pacchioni G. Trends in non-metal doping of anatase $\mathrm{TiO}_{2}: \mathrm{B}, \mathrm{C}, \mathrm{N}$ and $\mathrm{F}$. Catalysis Today. 2013;206:12-18. DOI: 10.1016/j.cattod.2011.11.030

[9] Han F, Kambala VSR, Srinivasan M, Rajarathnam D, Naidu R. Tailored titanium dioxide photocatalysts for the degradation of organic dyes in wastewater treatment: A review. Applied Catalysis A: General. 2009;359:25-40. DOI: 10.1016/j.apcata.2009.02.043

[10] Bouras P, Stathatos E, Lianos P. Pure versus metal-ion-doped nanocrystalline titania for photocatalysis. Applied Catalysis B: Environmental. 2007;73:51-59. DOI: 10.1016/j. apcatb.2006.06.007

[11] Choi W, Termin A, Hoffmann MR. The role of metal ion dopants in quantum-sized $\mathrm{TiO}_{2}$ : Correlation between photoreactivity and charge carrier recombination dynamics. Journal of Chemical Physics. 1994;98:13669-13679. DOI: 10.1021/j100102a038

[12] Wang Y, Zhang R, Li J, Li L, Lin S. First-principles study on transition metal-doped anatase $\mathrm{TiO}_{2}$. Nanoscale Research Letters. 2014;9:46. DOI: 12. 10.1186/1556-276X-9-46

[13] Bahers TL, Rérat M, Sautet P, Lyon UD, Claude U, Lyon B, et al. Semiconductors used in photovoltaic and photocatalytic devices: Assessing fundamental properties from DFT. Journal of Physical Chemistry C. 2014;118:5997-6008. DOI: 13. 10.1021/jp409724c

[14] Carter EA, Liaw P. New concepts and modeling strategies to design and evaluate photoelectro-catalysts based on transition metal oxides. Chemical Society Reviews. 2013;42: 2401-2422. DOI: $10.1039 / \mathrm{c} 2 \mathrm{cs} 35267 \mathrm{~b}$

[15] Norskov JK, Bligaard T, Rossmeisl J, Christensen CH. Towards the computational design of solid catalysts. Nature Chemistry. 2009;1:37-46. DOI: 10.1038/nchem.121

[16] Luttrell T, Halpegamage S, Tao J, Kramer A, Sutter E, Batzill M. Why is anatase a better photocatalyst than rutile?-Model studies on epitaxial $\mathrm{TiO}_{2}$ films. Scientific Reports. 2014;4:4043. DOI: 16. 10.1038/srep04043

[17] Zhang J, Zhou P, Liu J, Yu J. New understanding of the difference of photocatalytic activity among anatase, rutile and brookite $\mathrm{TiO}_{2}$. Physical Chemistry Chemical Physics. 2014;16:20382-20386. DOI: 10.1039/C4CP02201G

[18] Miglio A, Waroquiers D, Rignanese G-M, Gonze X. How does chemistry influence electron effective mass in oxides? A high-throughput computational analysis. Chemistry of Materials. 2014;26. DOI: 10.1021/cm404079a

[19] Jing T, Dai Y, Ma X, Wei W, Huang B. Electronic structure and photocatalytic watersplitting properties of $\mathrm{Ag}_{2} \mathrm{ZnSn}\left(\mathrm{S}_{1}{ }^{-}{ }_{\mathrm{x}} \mathrm{Se}_{\mathrm{x}}\right)_{4}$. The Journal of Physical Chemistry C. 2015;119:27900-27908. DOI: 10.1021/acs.jpcc.5b09522 
[20] Burke K. Perspective on density functional theory. The Journal of Chemical Physics. 2012;136(15):150901-150909. DOI: 10.1063/1.4704546

[21] Zhang IY, Su NQ, Brémond ÉAG, Adamo C, Xu X. Doubly hybrid density functional xDH-PBE0 from a parameter-free global hybrid model PBE0. The Journal of Chemical Physics. 2012;136(17):174103-174110. DOI: 10.1063/1.3703893

[22] Hautier G, Jain A, Ong S. From the computer to the laboratory: Materials discovery and design using first-principles calculations. Journal of Materials Science. 2012;47(21):73177340. DOI: $10.1007 / \mathrm{s} 10853-012-6424-0$

[23] Atkins P, de Paula J, Friedman R. Quanta, Matter and Change: A Molecular Approach to Physical Chemistry. New York: W. H. Freeman and Company; 2009. p. 986

[24] Lewars EG. Computational Chemistry. Introduction to the Theory and Applications of Molecular and Quantum Mechanics. 2ª Edição ed. Londres: Springer; 2011. p. 665

[25] Morgon NH, Custodio R. Teoria do Funcional de Densidade. Química Nova. 1995;18 (1):45-55

[26] Janesko BG, Henderson TM, Scuseria GE. Screened hybrid density functionals for solidstate chemistry and physics. Physical Chemistry Chemical Physics. 2009;11(3):443-454. DOI: 10.1039/B812838C

[27] Becke AD. Perspective: Fifty years of density-functional theory in chemical physics. The Journal of Chemical Physics. 2014;140:18A301. DOI: 10.1063/1.4869598

[28] Zhang IY, Wu J, Xu X. Extending the reliability and applicability of B3LYP. Chemical Communications. 2010;46:3057-3070. DOI: 10.1039/C000677G

[29] Perdew JP, Yue W. Accurate and simple density functional for the electronic exchange energy: Generalized gradient approximation. Physical Review B. 1986;33(12):8800-8802. DOI: 29. 10.1103/PhysRevB.33.8800

[30] Gunnarsson O, Jones RO. Total-energy differences: Sources of error in local-density approximations. Physical Review B. 1985;31(12):7588-7602. DOI: 30. 10.1103/PhysRevB.31.7588

[31] Becke AD. Density-functional thermochemistry. III. The role of exact exchange. Journal of Chemical Physics. 1993;98(7):5648-5652. DOI: 10.1063/1.464913

[32] Becke AD. Density-functional exchange-energy approximation with correct asymptotic behavior. Physical Review A. 1988;38(6):3098-3100. DOI: 32. 10.1103/PhysRevA.38.3098

[33] Kullgren J, Castleton CWM, Müller C, Ramo DM, Hermansson K. B3LYP calculations of cerium oxides. The Journal of Chemical Physics. 2010;132(5):054110. DOI: 10.1063/1.3253795

[34] Zhang Y, Xu X, Goddard WA. Doubly hybrid density functional for accurate descriptions of nonbond interactions, thermochemistry, and thermochemical kinetics. Proceedings of the National Academy of Sciences. 2009;106(13):4963-4968. DOI: 10.1073/pnas.0901093106 
[35] Stephens PJ, Devlin FJ, Chabalowski CF, Frisch MJ. Ab initio calculation of vibrational absorption and circular dichroism spectra using density functional force fields. The Journal of Chemical Physics. 1994;98(45):11623-11627. DOI: 10.1021/j100096a001

[36] Perdew JP, Ernzerhof M, Burke K. Rationale for mixing exact exchange with density functional approximations. The Journal of Chemical Physics. 1996;105(22):9982-9985. DOI: 36. 10.1063/1.472933

[37] Adamo C, Barone V. Toward reliable density functional methods without adjustable parameters: The PBE0 model. The Journal of Chemical Physics. 1999;110(13):6158-6170. DOI: $10.1063 / 1.478522$

[38] Henderson TM, Paier J, Scuseria GE. Accurate treatment of solids with the HSE screened hybrid. Physica Status Solidi (b). 2011;248(4):767-774. DOI: 10.1002/pssb.201046303

[39] West AR. Basic Solid State Chemistry. 2nd ed. Chichester: John Wiley \& Sons; 2006. p. 480

[40] Kwok HL. Electronic Materials. Boston: PWS Publishing Company; 1997

[41] Smart LE. Solid State Chemistry: An Introduction. 3rd ed. Boca Raton: Taylor \& Francis; 2005. p. 407

[42] Sutton AP. Eletronic Structure of Materials. Oxford: Oxford University Press; 1996. p. 260

[43] Rezende SM. A física de materiais e Dispositivos eletrônicos. 2nd ed. São Paulo: Editora Livraria da Física; 2004. p. 531

[44] Kuno M. Introductory Nanoscience: Physical and Chemical Concept. Oxford: Garland Science, Taylor \& Francis Group; 2011. p. 420

[45] Yu J, Zhou P, Li Q. New insight into the enhanced visible-light photocatalytic activities of B-, C- and B/C-doped anatase $\mathrm{TiO}_{2}$ by first-principles. Physical Chemistry Chemical Physics. 2013;15(29):12040-12047. DOI: 10.1039/C3CP44651D

[46] Zhou P, Yu J, Wang Y. The new understanding on photocatalytic mechanism of visiblelight response NS codoped anatase $\mathrm{TiO}_{2}$ by first-principles. Applied Catalysis B: Environmental. 2013;142-143:45-53. DOI: 10.1016/j.apcatb.2013.04.063

[47] Ma X, Dai Y, Guo M, Huang B. The role of effective mass of carrier in the photocatalytic behavior of silver halide-based $\mathrm{Ag} @ \mathrm{AgX}(\mathrm{X}=\mathrm{Cl}, \mathrm{Br}, \mathrm{I})$ : A theoretical study. Chemical Physics. 2012;13(9):2304-2309. DOI: 10.1002/cphc.201200159

[48] Soares GB, Ribeiro RAP, de Lazaro SR, Ribeiro C. Photoelectrochemical and theoretical investigation of the photocatalytic activity of $\mathrm{TiO}_{2}$ : N. RSC Advances. 2016;6(92):8968789698. DOI: 10.1039/C6RA15825K

[49] Keith JA, Anton J, Kaghazchi P, Jacob T. Modeling catalytic reactions on surfaces with density functional theory. In: Modeling and Simulation of Heterogeneous Catalytic Reactions. Wiley-VCH Verlag GmbH \& Co. KGaA; Weinheim, Germany, 2011. pp. 1-38. DOI: 10.1002/9783527639878.ch1 
[50] Nørskov JK, Abild-Pedersen F, Studt F, Bligaard T. Density functional theory in surface chemistry and catalysis. Proceedings of the National Academy of Sciences. 2011;108 937943. DOI: 10.1073/pnas.1006652108

[51] Cheng J, Sulpizi M, VandeVondele J, Sprik M. Hole localization and thermochemistry of oxidative dehydrogenation of aqueous rutile $\mathrm{TiO}_{2}(110)$. ChemCatChem. 2012;4:636-640. DOI: $10.1002 /$ cctc.201100498

[52] Migani A, Blancafort L. Excitonic interfacial proton-coupled electron transfer mechanism in the photocatalytic oxidation of methanol to formaldehyde on $\mathrm{TiO}_{2}$ (110). Journal of the American Chemical Society. 2016;138:16165-16173. DOI: 10.1021/jacs.6b11067

[53] Nolan M, Iwaszuk A, Lucid AK, Carey JJ, Fronzi M. Design of novel visible light active photocatalyst materials: Surface modified $\mathrm{TiO}_{2}$. Advanced Materials. 2016;28:5425-5446. DOI: 10.1002/adma.201504894 
